\title{
Comparing 3C 120 jet emission at small and large scales
}

\author{
D. Zargaryan ${ }^{1,2}$, S. Gasparyan ${ }^{1,2}$, V. Baghmanyan ${ }^{1,2,3}$, and N. Sahakyan ${ }^{2}$ \\ 1 ICRANet, Piazza della Repubblica 10, 65122 Pescara, Italy \\ e-mail: narek@icra.it \\ 2 ICRANet-Armenia, Marshall Baghramian Avenue 24a, 0019 Yerevan, Republic of Armenia \\ 3 Yerevan State University, Alek Manukyan str. 1, 0025 Yerevan, Republic of Armenia
}

Received 19 July 2017 / Accepted 15 September 2017

\begin{abstract}
Context. Important information on the evolution of a jet can be obtained by comparing the physical state of the plasma at its propagation through the broad-line region (where the jet is most likely formed) into the intergalactic medium, where it starts to decelerate significantly.

Aims. We compare the constraints on the physical parameters in the innermost $(\leq \mathrm{pc})$ and outer $(\geq \mathrm{kpc})$ regions of the $3 \mathrm{C} 120$ jet by means of a detailed multiwavelength analysis and theoretical modeling of their broadband spectra.

Methods. The data collected by Fermi LAT ( $\gamma$-ray band), Swift (X-ray and ultraviolet bands), and Chandra (X-ray band) are analyzed together and the spectral energy distributions are modeled using a leptonic synchrotron and inverse Compton model, taking into account the seed photons originating inside and outside the jet. The model parameters are estimated using the Markov chain Monte Carlo method.

Results. The $\gamma$-ray flux from the inner jet of 3C 120 was characterized by rapid variation from MJD 56900 to MJD 57300 . Two strong

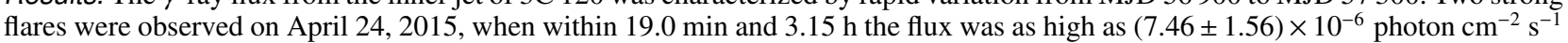
and $(4.71 \pm 0.92) \times 10^{-6}$ photon $\mathrm{cm}^{-2} \mathrm{~s}^{-1}$, respectively, with $\geq 10 \sigma$. During these flares the apparent isotropic $\gamma$-ray luminosity was $L_{\gamma} \simeq(1.20-1.66) \times 10^{46} \mathrm{erg} \mathrm{s}^{-1}$ which is not common for radio galaxies. The broadband emission in the quiet and flaring states can be described as synchrotron self-Compton emission, while inverse Compton scattering of dusty torus photons cannot be excluded for the flaring states. The X-ray emission from the knots can be reproduced by inverse Compton scattering of cosmic microwave background photons only if the jet is highly relativistic (even when $\delta=10, U_{\mathrm{e}} / U_{\mathrm{B}}$ is still $\geq 80$ ). These extreme requirements can be somewhat softened assuming the $\mathrm{X}$-rays are from the synchrotron emission of a second population of very high energy electrons.

Conclusions. We found that the jet power estimated at two scales is consistent, suggesting that the jet does not suffer severe dissipation, it simply becomes radiatively inefficient.
\end{abstract}

Key words. galaxies: individual: 3C 120 - gamma rays: galaxies - X-rays: galaxies - radiation mechanisms: non-thermal

\section{Introduction}

The recent observations in the high energy (HE; $\geq 100 \mathrm{MeV}$ ) $\gamma$-ray band show that the extragalactic $\gamma$-ray sky is dominated by the emission of active galactic nuclei (AGNs) of different types. The dominant type is blazars, an extreme class of AGNs with jets that form a small angle with respect to the line of sight (Urry \& Padovani 1995). Blazars are very bright and luminous sources known to emit electromagnetic radiation in almost all frequencies that are currently being observed, ranging from radio to very high energy (VHE; $>100 \mathrm{GeV}) \gamma$-ray bands. Their broadband spectrum is mainly dominated by nonthermal emission produced in a relativistic jet pointing toward the observer. Another important class of $\gamma$-ray emitting AGNs observed by Fermi Large Area Telescopes (Fermi LAT) are radio galaxies with relativistic jets at systematically larger angles (Abdo et al. 2010a; Kataoka et al. 2011). Due to larger jet inclination angles compared with those of blazars, the jet emission is not significantly Doppler boosted, making it less prevalent over such components as the radiation from mildly relativistic outflows or emission from extended structures. This opened a new window onto the particle acceleration and emission processes in different components of AGNs. The radio galaxy 3C 120, at a distance of $\approx 144.9 \mathrm{Mpc}$, is an active and powerful emitter in all the observed wavebands. In the radio band, its characteristics are closer to the Fanaroff-Riley class I radio sources (Fanaroff \& Riley 1974) with a powerful one-sided radio jet from sub-pc to $100 \mathrm{kpc}$ scales (Walker et al. 1987). The one-sided parsec-scale jet has been studied by long baseline interferometry, and superluminal motion has been observed with apparent speed up to 4-6 $c$ (Homan et al. 2001; Gómez et al. 1998, 1999). Recently, using X-ray and radio observations, Marscher et al. (2002) found that the dips in the X-ray emission are followed by ejections of bright superluminal knots in the radio jet which clearly establishes an accretion disk-jet connection. The kpc-scale jet of 3C 120 has a complex structure with several knots, k4, k7, s2, s3, and k25 (see Fig. 2 in Harris et al. 2004, where on the $1.6 \mathrm{GHz}$ radio contours the section of the jet with knot labels is shown), detected in the radio, optical, and X-ray bands (Harris et al. 1999, 2004). The knots are labeled by their distance from the core in arcseconds (e.g., k4, k7) and the smooth sections of the jet detected in the optical band (Hjorth et al. 1995) are labeled s2 and s3. These knots appeared to have interesting morphology and spectra, the most tricky of which is the X-ray emission from the knot k25: it has a very weak radio flux, but it is bright in the $\mathrm{X}$-ray band. It is a real challenge for the one-zone synchrotron 
emission scenario to interpret the emission from $\mathrm{k} 25$, in which case a large deviation from the minimal energy condition is required. It has been suggested that $\mathrm{X}$-rays might be produced through the synchrotron radiation of an electron population distinct from that responsible for the radio emission (Harris et al. 2004). Alternative theories such as proton synchrotron emission (Aharonian 2002) or inverse-Compton scattering of CMB photons (Zhang et al. 2010) have also been proposed. However, to date it is not clear which is the exact mechanism responsible for the X-ray emission.

The core of the 3C 120 jet itself has interesting and peculiar features. It is very bright in the X-ray band with a flux of $\approx 5 \times 10^{-11} \mathrm{erg} \mathrm{cm}^{-2} \mathrm{~s}^{-1}$ at $2-10 \mathrm{keV}$, variable on time scales from days to months (Halpern 1985). The $\gamma$-rays from 3C 120 had been already detected by Fermi LAT during first 15 months' scan of the whole sky (Abdo et al. 2010a), which was then confirmed by the data accumulated for two years (Kataoka et al. 2011). In addition, long-term variability (several months) had been found using the five-year Fermi LAT data (Sahakyan et al. 2015) with short periods (days and hours) of brightening (Tanaka et al. 2015; Janiak et al. 2016). Inverse Compton scattering of synchrotron photons seems to be the mechanism responsible for the $\gamma$-ray emission from 3C 120 (Tanaka et al. 2015; Sahakyan et al. 2015), while the flares and the fast $\gamma$-ray variability are explained within more complex structured jet scenarios (Janiak et al. 2016).

Combining the data derived at the sub-pc and kilo-parsec regions of the same jet could greatly help to understand the features of powerful extragalactic jets; for example, it could shed some light on the evolution and propagation of the jets from the central engine to the outer regions where the jet is starting to significantly decelerate. This approach can be fruitfully applied to the sources showing a large-scale jet long enough to be resolved by Chandra. Unfortunately, the best-studied blazars tend not to have well-studied large-scale jets, precisely because the blazars are most closely aligned with the line of sight, reducing the projected angular dimension of the large-scale jet. Thus, only a few jets can be studied on both scales. The prominent features of $3 \mathrm{C} 120$, i.e., the strong jet well resolved in both small (pc) and large (kpc) scales, makes this object an ideal target for investigation of the processes occurring in the powerful jet along its propagation.

The low statistics in the $\gamma$-ray band did not allow us to study the flux changes on submonthly time scales (the light curves contain many upper limits). The recent update of the Fermi LAT event-level analysis from PASS7 to PASS8 has significantly improved the event reconstruction and classification, which increased the sensitivity and improved the angular resolution. Combining this with the data accumulated for a longer period ( $8 \mathrm{yr}$ ) would significantly improve the statistics and would allow us to perform a detailed study of the $\gamma$-ray flux evolution over time. Also, the analysis of the Swift data will allow us to explore the emission from the core region with the help of contemporaneous spectral energy distributions (SEDs). Moreover, several Chandra observations of the large-scale jet of 3C 120 in 2001-2016 provide an overall exposure that is large enough to perform a spectral analysis of the X-ray data. This motivated us to have a new look at the emission from the jet of 3C 120 in small and large scales using the most recent data available.

The paper is structured as follows. The analyses of Fermi LAT and Swift XRT/UVOT data are presented in Sect. 2. The analysis of Chandra data is described in Sect. 3. The modeling of the emission from the core and knots is presented in Sect. 4. The results are discussed in Sect. 5 and summarized in Sect. 6.

\section{Observations and data analysis of central region}

\subsection{Fermi LAT data extraction}

On board the Fermi satellite, LAT is a pair-conversion telescope designed to detect $\mathrm{HE} \gamma$-rays in the energy range $20 \mathrm{MeV}-$ $300 \mathrm{GeV}$. Operating since August 4, 2008, it is always in the survey mode by default, scanning the entire sky every $\sim 3 \mathrm{~h}$ and thereby providing continuous monitoring of $\gamma$-ray sources. Details about the Fermi LAT can be found in Atwood et al. (2009).

In the current paper we analyze the data accumulated over $8 \mathrm{yr}$, from the beginning of the Fermi LAT mission up to August 8, 2016 (MET 239557417-460339204). Fermi LAT Science Tool version v10r0p5 is used with the instrument response function P8R2_SOURCE_V6. The recently updated PASS8 version of the data in the energy range between $100 \mathrm{MeV}-300 \mathrm{GeV}$ is analyzed. The entire data set is filtered with gtselect and gtmktime tools and only the events with a high probability of being photons evclass $=128$, evtype $=3$ have been considered. The zenith angle cutoff $>90^{\circ}$ is made to exclude atmospheric $\gamma$-rays from the Earth limb that can be a significant source of background.

The photons from a circular region of interest (ROI) centered on VLBI radio positions of 3C 120 (RA, Dec) $=(68.296$, $5.354)$ are used in the analysis. The photons are binned within $14.1^{\circ} \times 14.1^{\circ}$ square regions with gtbin tool, with a stereographic projection into $0.1^{\circ} \times 0.1^{\circ}$ pixels. In order to account for the emission from other sources within the ROI, the model file is generated using the Fermi-LAT third source catalog (Acero et al. 2015 ) and the sources within $10^{\circ}+5^{\circ}$ of the position of $3 \mathrm{C} 120$ are included in the model file. Since $3 \mathrm{C} 120$ is not included in the catalog, a point-like source in the known location of 3C 120 was added to the model file. The Galactic background component is modeled using the LAT standard diffuse background model $\mathrm{gll}$ iem _ v05_rev1 and iso_source_v05 - for the isotropic $\gamma$-ray background. The normalization of background models as well as the fluxes and spectral indices of the sources within $10^{\circ}$ are left as free parameters during the analysis.

\subsection{Temporal variability}

In order to have SEDs with contemporaneous data for broadband modeling we created $\gamma$-ray light curves with different time binning. The $\gamma$-ray light curve is calculated with the unbinned likelihood analysis method implemented in the gtlike tool. Photons in the energy range of $(0.1-300) \mathrm{GeV}$ are used in the analysis with the appropriate quality cuts applied in the data selection. The photon indices of all background sources are fixed to the best guess values obtained in full time analysis in order to reduce the uncertainties in the flux estimations. The power-law index of 3C 120 is first considered as a free parameter and then as a fixed one. Since no variability is expected for the background diffuse emission, the normalization of both background components is also fixed to the values obtained for the whole time period.

The light curve obtained for 90-day binning is shown in the upper panel of Fig. 1. Before $\approx$ MJD 56000 (March 14, 2012), the source is mostly undetectable by Fermi LAT; only in two of the total 15 cases did the source detection significance exceed the required threshold of $4 \sigma$. Then the source flux was high enough to be detected by Fermi LAT, and up to $\approx$ MJD 56800 it remained constant with no significant changes. Starting from $\approx$ MJD 56800, the flux substantially increased up to a few times $10^{-7}$ photon $\mathrm{cm}^{2} \mathrm{~s}^{-1}$ and remained so until $\approx$ MJD 57 350. The standard $\chi^{2}$ analysis revealed a highly variable $\gamma$-ray flux, where the probability that the flux is constant is $p\left(\chi^{2}\right) \ll 5 \%$. No strong 


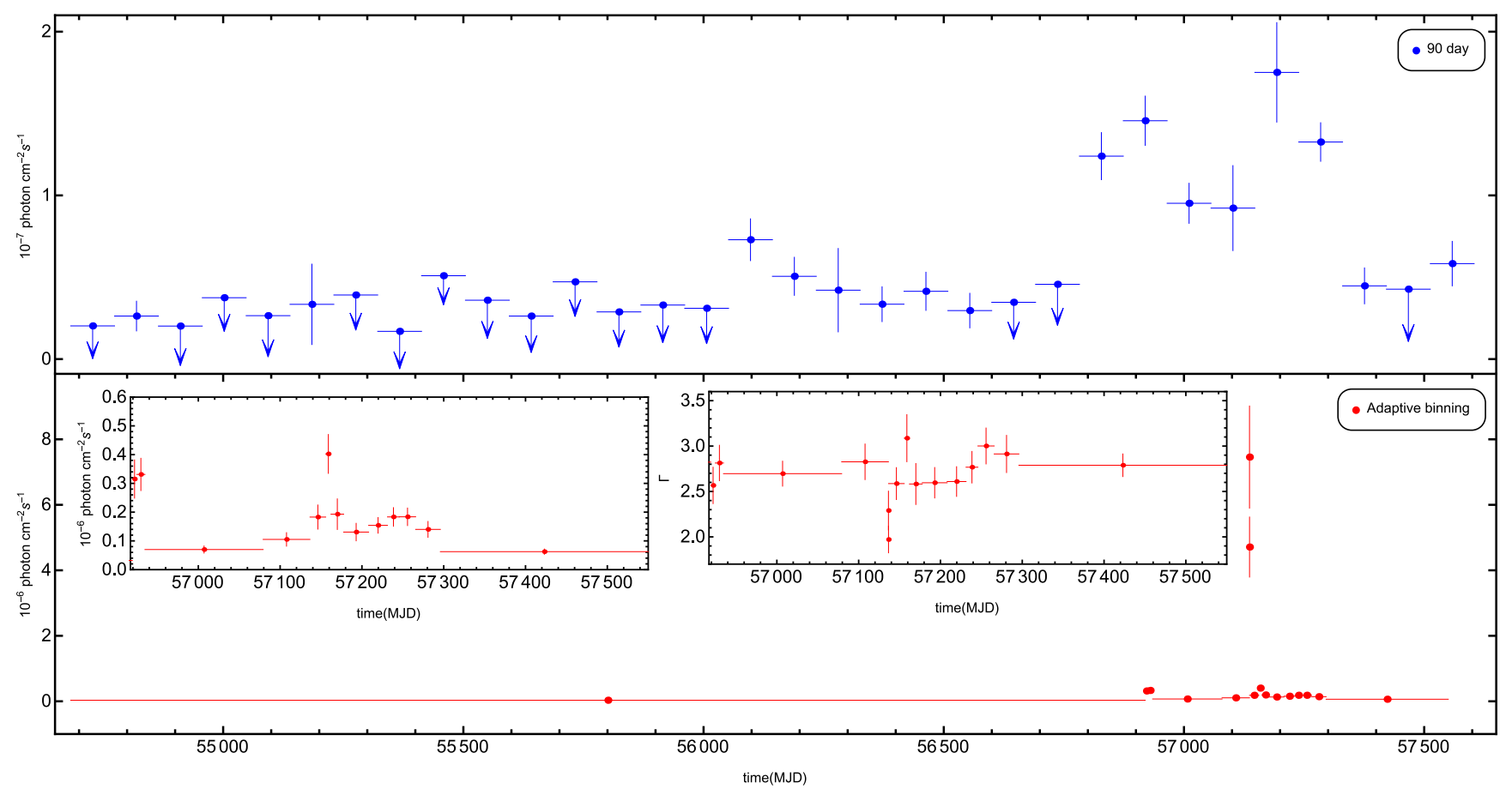

Fig. 1. Gamma-ray light curve of 3C 120 from August 4, 2008, to August 4, 2016. Panel $a$ : bin intervals corresponding to 90 days. Panel b: light curve obtained by adaptive binning method assuming $20 \%$ uncertainty. The change in photon index is shown in the insert.

variation in the $\gamma$-ray photon index is found during the time under consideration.

The active state identified above can be further investigated using denser time sampling. However, considering the relatively weak flux, the light curve will contain many upper limits that prevent us from making any conclusions. Therefore, a light curve generated with an adaptive binning method is used. In this method, the time bin widths are flexible and are chosen such that they produce bins with constant flux uncertainty (Lott et al. 2012). This method allows detailed investigation of flux changes in time since at times of high fluxes the time bins are narrower than during lower ones, therefore allowing the rapid changes of the fluxes to be found. In order to reach the necessary relative flux uncertainty, the integral fluxes are computed above the optimal energies Lott et al. (2012; $E_{0}=183.2 \mathrm{MeV}$ in this case). Also, in order to improve the accuracy of the method, the flux of bright sources that lie close to $3 \mathrm{C} 120$ have been taken into account. This is done by providing the parameters of confusing sources during the adaptive binning light curve calculations.

For $20 \%$ adaptively binned intervals, the light curve is generated for the energy range $100 \mathrm{MeV}-300 \mathrm{GeV}$ (lower panel in Fig. 1). As expected, initially it took a long time to reach the necessary $20 \%$ uncertainty. Indeed, the first bin contains the data from the start of the mission to MJD 56919.31 (19 September 2014), amounting to more than 6 yr. Afterwards, it took less time to reach the required uncertainty. The most dramatic increase in the $\gamma$-ray flux was observed on April 24, 2015. First, within 19.0 min the flux reached $(7.46 \pm 1.56) \times$ $10^{-6}$ photon $\mathrm{cm}^{-2} \mathrm{~s}^{-1}$ with $\Gamma=2.29 \pm 0.21$ and $11.2 \sigma$ detection significance. Then for another $3.15 \mathrm{~h}$ it was as high as $(4.71 \pm 0.92) \times 10^{-6}$ photon $\mathrm{cm}^{-2} \mathrm{~s}^{-1}$ with $\Gamma=1.97 \pm 0.14$ and $12.7 \sigma$. Then the flux slowly decreased to a few times $10^{-7}$ photon $\mathrm{cm}^{-2} \mathrm{~s}^{-1}$ with the bin size varying from 10 to 35 days. The source was in an active state up to $\approx$ MJD 57300 and then turned again into its quiescent state, in which case the data should be accumulated for $\simeq 254$ days.

\subsection{Spectral analysis}

In order to investigate the emission from 3C 120 in its quiet and active states, the $\gamma$-ray spectra were extracted from the following periods:

1) long quiescent states, namely between MJD 54682.65 and MJD 56919.31;

2) the active state after MJD 56919.31. The period overlaps with the Swift observations on MJD 56934.19, 56937.70, and 5638.50. Although the Swift observation lasted several thousands of seconds, in order to increase the $\gamma$-ray photon statistics, the Fermi LAT spectrum was extracted from 15 days (MJD 56 919.31-56 934.76) when the source showed a comparable flux level (as inferred from the light curve obtained by an adaptive binning method).

The spectrum of 3C 120 was modeled as a power-law function $\left(\mathrm{d} N / \mathrm{d} E \sim N_{0} E^{-\Gamma}\right)$ with the normalization and index considered as free parameters. In order to find the best matches between the spectral models and events, a binned likelihood analysis is performed with gtlike for the first period, while an unbinned analysis was applied for the second one. The spectral fitting results are summarized in Table 1 and the plot of the SEDs is shown in Fig. 2. During the flaring periods the $\gamma$-ray flux increased by nearly an order of magnitude, and the photon index hardened.

\subsection{Swift observations}

Swift satellite (Gehrels et al. 2004) observed 3C 120 in its $\gamma$-ray quiescent and active states. As the X-ray flux varies as well ${ }^{1}$, we have selected the observations made on MJD 55252.70 and MJD 55800.25 when the X-ray flux was also low. During the $\gamma$-ray active state 3C 120 was observed only three times: on MJD 56934.19, MJD 56937.70 and MJD 56938.50. The data from two of the instruments on board Swift, the UltraViolet and

\footnotetext{
1 wwW.bu .edu/blazars/VLBA_GLAST/3c120.html
} 
Table 1. Parameters of spectral analysis.

\begin{tabular}{|c|c|c|c|}
\hline & Swift XRT & & \\
\hline \multirow[t]{2}{*}{ Obsid } & $\Gamma_{\mathrm{X}}$ & $v F_{v}$ & Reduced $\chi^{2}$ \\
\hline & MJD & $\times 10^{-11} \mathrm{erg} \mathrm{cm}^{-2} \mathrm{~s}^{-1}$ & \\
\hline 37594002 & $1.42 \pm 0.07$ & $4.27 \pm 0.18$ & 0.43 \\
\hline 37594004 & $1.53 \pm 0.08$ & $2.41 \pm 0.11$ & 0.75 \\
\hline 37594042 & $1.76 \pm 0.04$ & $6.73 \pm 0.15$ & 1.21 \\
\hline 37594048 & $1.72 \pm 0.04$ & $5.37 \pm 0.12$ & 1.05 \\
\hline \multirow[t]{2}{*}{37594049} & $1.80 \pm 0.06$ & $3.73 \pm 0.13$ & 0.86 \\
\hline & Fermi LAT & & \\
\hline \multirow[t]{2}{*}{ date } & Flux & $\Gamma$ & TS \\
\hline & $10^{-8}$ photon $\mathrm{cm}^{-2} \mathrm{~s}^{-1}$ & & \\
\hline $2008 / 08 / 04-2014 / 09 / 19$ & $2.87 \pm 0.49$ & $2.79 \pm 0.08$ & 179.43 \\
\hline \multirow[t]{2}{*}{ 2014/09/19-2014/10/04 } & $24.9 \pm 4.21$ & $2.57 \pm 0.16$ & 90.5 \\
\hline & Chandra & & \\
\hline \multirow[t]{2}{*}{ Region } & $\Gamma_{\mathrm{X}}$ & $v F_{v}$ & reduced $\chi^{2}$ \\
\hline & \multicolumn{3}{|c|}{$\times 10^{-14} \mathrm{erg} \mathrm{cm}^{-2} \mathrm{~s}^{-1}$} \\
\hline $\mathrm{k} 4$ & $1.82 \pm 0.10$ & $15.97 \pm 2.3$ & 1.03 \\
\hline k7 & $2.72 \pm 0.66$ & $1.85 \pm 0.82$ & 1.03 \\
\hline s2 & $2.64 \pm 1.26$ & $0.78 \pm 0.51$ & 0.81 \\
\hline s3 & $2.14 \pm 0.28$ & $0.45 \pm 0.37$ & 0.89 \\
\hline k25 inner & $1.63 \pm 0.22$ & $3.89 \pm 1.42$ & 0.98 \\
\hline k25 outer & $1.62 \pm 0.11$ & $12.16 \pm 1.9$ & 0.79 \\
\hline k25 new & $1.80 \pm 0.19$ & $6.28 \pm 1.67$ & 0.91 \\
\hline
\end{tabular}

Optical Telescope (UVOT) and the X-Ray Telescope (XRT), have been used in the analysis.

\subsection{Swift XRT}

The Swift -XRT observations were made in the photon counting (PC; Obsid 37594002, 37594004) and windowed timing (WT; Obsid 37594042, 37594048, 37594049) modes. The data were analyzed using the XRTDAS software package (v.3.3.0) distributed by HEASARC as part of the HEASoft package (v.6.21). The source spectrum region was defined as a circle with a radius of 30 pixels $\left(\sim 71^{\prime \prime}\right)$ at the center of the source, while the background region was defined as an annulus centered at the source with its inner and outer radii being $80\left(\sim 190^{\prime \prime}\right)$ and 120 pixels $\left(\sim 280^{\prime \prime}\right)$, respectively. For the PC-mode observation 37594004, the count rate was above 0.5 count/s because it was affected by the piling up in the inner part of the PSF. This effect was removed by excluding the events within a 4-pixel radius circle centered on the source position. Then, using xrtmkarf task, ancillary response files were generated by applying corrections for the PSF losses and CCD defects.

The spectrum was rebinned to have at least 20 counts per bin, ignoring the channels with energy below $0.5 \mathrm{keV}$, and fitted using XSPEC v12.9.1a. The results of the fit are given in Table 1 and the corresponding spectra are shown in Fig. 2. The $0.5-6.0 \mathrm{keV}$ spectrum is well fitted by an absorbed power-law model with column density $N_{\mathrm{H}}=1.06 \times 10^{21} \mathrm{~cm}^{-2}$. Although the X-ray flux did not increase significantly (the highest flux of $F_{0.5-6 \mathrm{keV}} \simeq(6.73 \pm 0.15) \times 10^{-11} \mathrm{erg} \mathrm{cm}^{-2} \mathrm{~s}^{-1}$ observed on October 4,2014 , exceeds the lowest by $\sim 2.8$ times), the X-ray photon index softened, changing in the range of $\Gamma_{\mathrm{x}}=(1.76-1.80)$ during the bright $\gamma$-ray periods.

\subsection{Swift UVOT}

In the analysis of the Swift UVOT data, the source counts were extracted from an aperture of 5.0" radius around the source. The background counts were taken from the neighboring circular region having a radius of $20^{\prime \prime}$. The magnitudes were computed using the uvotsource tool (HEASOFT v6.21), corrected for extinction according to Roming et al. (2009) using $E(B-V)=$ 0.256 from Schlafly \& Finkbeiner (2011) and zero points from Breeveld et al. (2011), converted to fluxes following Poole et al. (2008). The corresponding spectra are shown in Fig. 2. The optical-UV data points harden during the flaring periods.

\section{Chandra observations of the knots}

In the public archive we found five observations (ObsId 3015, $16221,17564,17565,17576)$ of 3C 120 with the Chandra telescope; the overall observation time was $251.86 \mathrm{ksec}$. We applied the standard data reduction procedure, using the Chandra Interactive Analysis of Observations (CIAO) 4.8 with Chandra Calibration Database (CALDB) version 4.7.2. We checked for flaring background events and did not find any significant flares. The readout streaks were removed for each observation and the events files were then re-projected to a single physical coordinate system (using observation 16221 as a reference). Also, to reduce the uncertainties caused by the position offsets of different observations, we made astrometric corrections, following the standard procedure for chandra data ${ }^{2}$. For each knot, the source region and the background are selected based on the position and shape given in Table 3 and Fig. 3 of Harris et al. (2004).

We extracted the spectra and created weighted response files for each observation, using the specextract script. The knots spectra were rebinned using a different count threshold depending on the total number of counts, and fitted in the $0.5-10 \mathrm{keV}$ energy range using a power law with the Galactic absorption model (column density fixed at $N_{\mathrm{H}}=1.06 \times 10^{21} \mathrm{~cm}^{-2}$ ) where the index and the normalization are allowed to vary freely. The spectral fit was done with Sherpa using the levmar optimization method and chi2datavar statistics.

The fitted spectral parameters are summarized in Table 1. The X-ray emission from the core is heavily saturated making it impossible to study the innermost parts of the jet. However, the nearby $\mathrm{k} 4$ knot emission is well resolved from the core; the $\mathrm{X}$-ray spectral index is $1.82 \pm 0.1$ and the flux $(1.60 \pm 0.23) \times$ $10^{-13} \mathrm{erg} \mathrm{cm}^{-2} \mathrm{~s}^{-1}$. The net counts from the $\mathrm{k} 7, \mathrm{~s} 2$, and $\mathrm{s} 3 \mathrm{knots}$ are relatively low $<50$ (compared with $>100$ from other knots), not enough for a detailed spectral fitting. The fitting resulted in a steep X-ray slope $(>2.0)$ and a relatively faint X-ray flux $\left(\leq 10^{-14} \mathrm{erg} \mathrm{cm}^{-2} \mathrm{~s}^{-1}\right)$. Because of low statistics and estimated large uncertainties, we did not consider them further. Following Harris et al. (2004) we also subdivide k25 into inner, outer, and new regions that have different properties in the radio band (the inner refers to the upstream edge and the outer refers to the western edge, see Fig. 3 of Harris et al. 2004). The X-ray emission from all three regions has harder X-ray emission spectra $(\leq 1.8)$ with the $X$-ray flux varying within $(3.89-12.16) \times$ $10^{-14} \mathrm{erg} \mathrm{cm}^{-2} \mathrm{~s}^{-1}$. It is interesting to note that the flux of k25 outer is at the same level as that of the bright $\mathrm{k} 4$, but with a significantly harder X-ray spectral index of $\Gamma_{X}=1.62 \pm 0.11$. The knot SEDs shown in Fig. 3 have been calculated by sample_energy_flux in Sherpa.

\footnotetext{
2 www.cxc.harvard.edu/ciao/threads/reproject_aspect/
} 

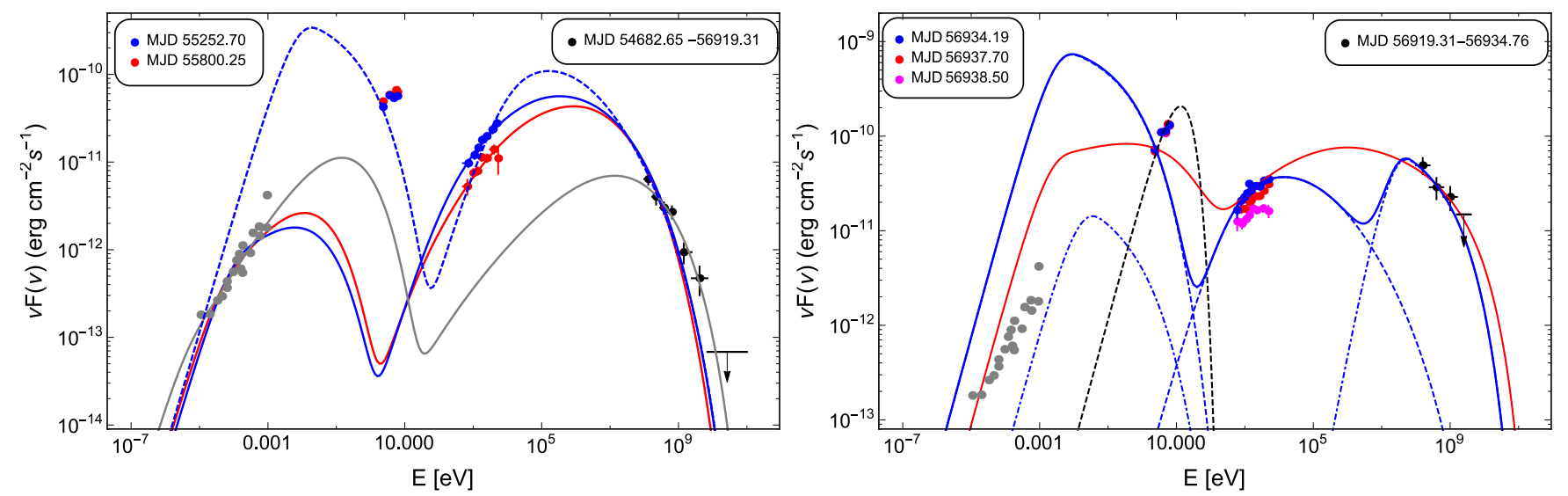

Fig. 2. Broadband SED of 3C 120 core emission for quiescent (left) and flaring (right) states. Left panel: blue and red solid lines are the synchrotron/SSC model fitting for two different X-ray fluxes, taking into account the radio data and assuming Swift UVOT data are upper limits. Instead the dashed blue line is calculated assuming that optical/UV emission is also produced by the jet. Right panel: SED in flaring state fitting with one-zone synchrotron/SSC (red solid line) and two-zone SSC+ EIC (blue solid line) models. The model parameters are presented in Table 2.
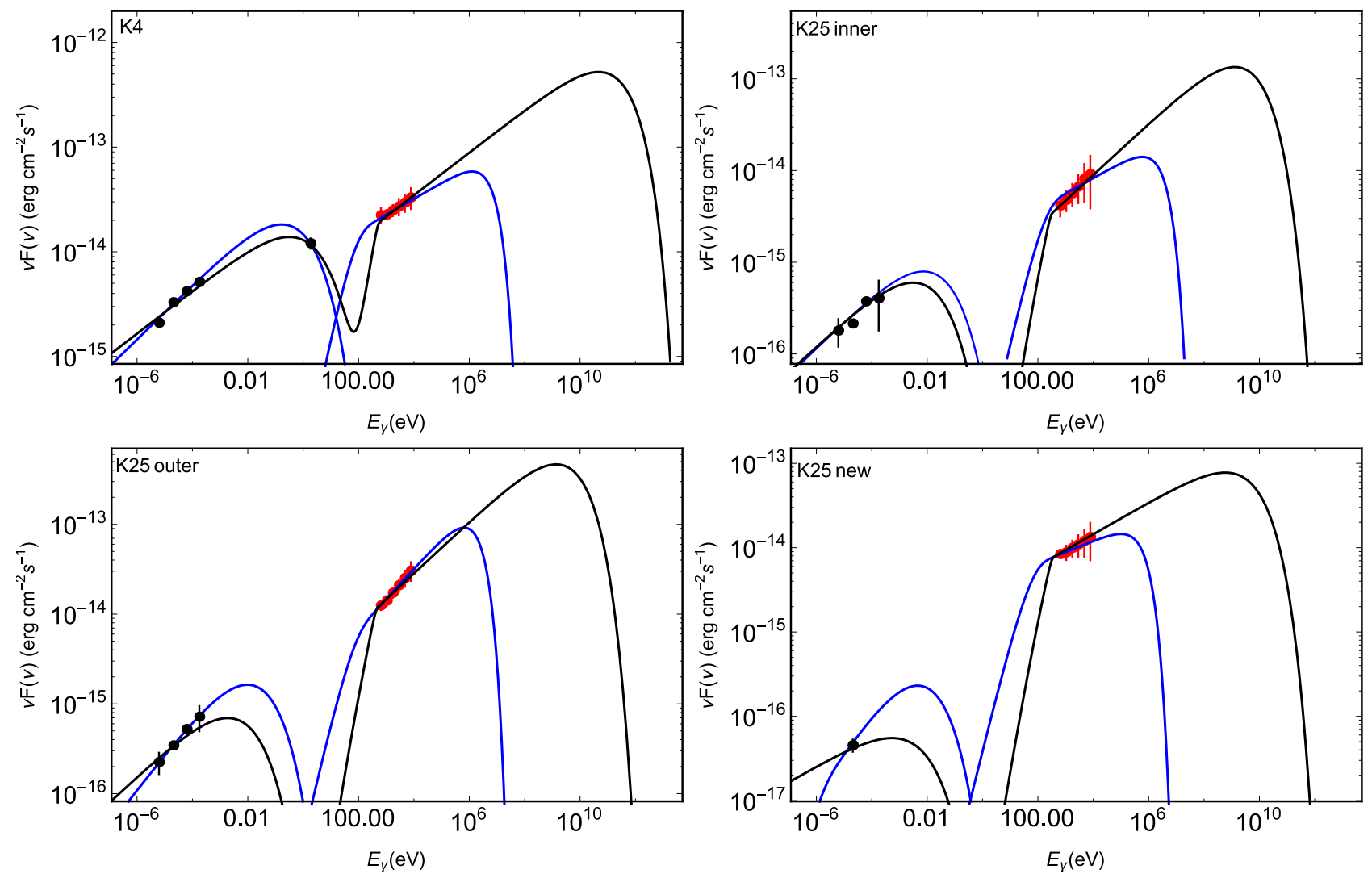

Fig. 3. SED of knots. The radio to optical data (black points) are from previous studies and the X-ray data (red points) are derived in this paper. The black lines are the IC/CMB model calculated for $\delta=10$ and the blue lines are the fit by the two-component synchrotron model.

\section{Modeling the spectral energy distributions}

\subsection{The core region}

The broadband SEDs of the 3C 120 core emission in its quiescent and flaring states are shown in Fig. 2 with the radio data (gray) from (Giommi et al. 2012; Tanaka et al. 2015) where the data from the period corresponding to the $\gamma$-ray quiescent state of 3C 120 is analyzed. As in the previous studies (Giommi et al. 2012; Sahakyan et al. 2015; Tanaka et al. 2015; Janiak et al. 2016) the SEDs hint at the existence of two nonthermal emission peaks in the IR/optical/UV and HE $\gamma$-ray bands. The UVOT data revealed a rather hard optical-UV spectrum which indicates that perhaps direct thermal emission from the accretion disk was being observed (Ghisellini et al. 2009a; Ghisellini \& Tavecchio 2009; Ghisellini et al. 2011).

Taking into account the results of the previous studies of other Fermi LAT-observed radio galaxies (Abdo et al. 2009, 2010b; Sahakyan et al. 2015; Tanaka et al. 2015), the multiwavelength emission of $3 \mathrm{C} 120$ is modeled using the synchrotron self-Compton (SSC; Maraschi et al. 1992; Bloom \& Marscher 1996; Ghisellini et al. 1985) and/or external inverse-Compton (EIC; Sikora et al. 1994) models. The radio 
through optical emission is due to the synchrotron emission of energetic electrons in the homogeneous randomly oriented magnetic field, while the X-ray to HE $\gamma$-ray emission is due to the inverse Compton scattering of the same synchrotron photons or photons of external origin.

In the flaring state (right panel of Fig. 2) the X-ray flux moderately increased and the spectrum softened, while the HE $\gamma$-ray flux increased and its spectrum shifted to higher energies. Within the synchrotron/SSC or EIC scenarios these modifications can be explained as the result of introducing changes in the electron acceleration, increasing the comoving radius and bulk Lorentz factor, or including the contribution from external photons (Paggi et al. 2011). Here we discuss the following possibilities: (i) in the quiescent state the jet energy dissipation occurs close to the central black hole and the dominant mechanism is the synchrotron/SSC emission; (ii) in the flaring period again the dominant processes is SSC, although the emission region has a higher bulk Lorentz factor; and (iii) the optical/UV/X-ray emission is due to the synchrotron/SSC emission from an extended and slow-moving region, while the HE $\gamma$-rays come from a compact and fast-moving region where EIC dominates. This is similar to the scenario adopted by Tavecchio et al. (2011) to explain the very fast VHE $\gamma$-ray variations and the hard GeV spectrum of PKS $1222+216$. The choice of this scenario is justified since strong changes are observed only in the $\gamma$-ray band.

The emission region (the "blob") is assumed to be a sphere with radius $R$ that carries a magnetic field with an intensity $B$ and a population of relativistic electrons that have a power law with an exponential cutoff energy distribution expected from shock acceleration theories (Inoue \& Takahara 1996):

$N_{\mathrm{e}}^{\prime}\left(E_{\mathrm{e}}^{\prime}\right)=N_{0}^{\prime}\left(E_{\mathrm{e}}^{\prime} / m_{\mathrm{e}} c^{2}\right)^{-\alpha} \exp \left[-E_{\mathrm{e}}^{\prime} / E_{\text {cut }}^{\prime}\right]$

for $E_{\min }^{\prime} \leq E_{\mathrm{e}}^{\prime} \leq E_{\max }^{\prime}$, where $E_{\min }^{\prime}$ and $E_{\max }^{\prime}$ are the minimum and maximum electron energies, respectively. The total electron energy $U_{\mathrm{e}}=\int_{\mathrm{E}_{\min }^{\prime}}^{\mathrm{E}_{\max }^{\prime}} E_{\mathrm{e}}^{\prime} N_{\mathrm{e}}^{\prime}\left(E_{\mathrm{e}}^{\prime}\right) \mathrm{d} E_{\mathrm{e}}^{\prime}$ is defined by $N_{0}^{\prime}, \alpha$ is the electron spectral index, and $E_{\text {cut }}^{\prime}$ is the cutoff energy.

Since the blob moves along the jet with a bulk Lorentz factor of $\Gamma_{\text {bulk }}$, the radiation will be amplified by a relativistic Doppler factor of $\delta=1 / \Gamma_{\text {bulk }}\left(1-\beta \cos \left(\theta_{\text {obs }}\right)\right)$, where $\theta_{\text {obs }}$ is the angle between the bulk velocity and the line of sight. For $3 \mathrm{C} 120$, the averaged bulk Lorentz factor has been estimated to be $\Gamma_{\text {bulk }}=$ $5.3 \pm 1.2$ (Jorstad et al. 2005; Casadio et al. 2015), while different mean values for $\theta_{\text {obs }}$ were obtained in VLBI observations; $\theta_{\text {obs }}$ varies from $9.7^{\circ}$ (Hovatta et al. 2009) to $20.5^{\circ}$ (Jorstad et al. 2005). So, we assumed $\delta=4$ (e.g., $\left.\theta_{\text {obs }} \sim\left[15^{\circ}-20^{\circ}\right]\right)$ and $\delta \approx 6$ (e.g., $\theta_{\text {obs }} \sim 9.7^{\circ}$ ) for the quiescent and flaring states, respectively. In the quiescent state, since no significant $\gamma$-ray variability is observed (or it varies in a long period) most likely the emission is produced in a large region for which we assume $R \approx$ $4 \times 10^{17} \mathrm{~cm} \sim 0.1 \mathrm{pc}$. Instead, in the active state the fast $\gamma$-ray flares in day/subday time scales indicate that the emitting region size should be $R / \delta \leq c \times t \times \delta=1.56 \times 10^{16}$ ( $t / 1$ day) $(\delta / 6) \mathrm{cm}$.

In the flaring state, the inverse Compton scattering of external photons either reflected from the broad line region (BLR; Sikora et al. 1994) or from the hot dusty torus (Błażejowski et al. 2000; Ghisellini et al. 2009b) can contribute to the emission in the $\gamma$-ray band. For any reasonable assumption about the jet opening angle $\left(\theta=0.1^{\circ}\right)$ and Doppler boosting factor $(\delta=6)$ the $\gamma$-ray emission region is at the distance $\sim R / \theta \approx 2.3 \mathrm{pc}$, well beyond the radius of $\operatorname{BLR}(5.9-7.4) \times 10^{16} \mathrm{~cm}$ determined from reverberation mapping (Pozo Nuñez et al. 2014). In this case the dominant external photon field is the IR radiation from the hot dusty torus, which we assume has a blackbody spectrum with a luminosity of $L_{\mathrm{IR}}=\eta L_{\mathrm{disk}}(\eta=0.6)$ (Ghisellini \& Tavecchio 2009) and these photons are filling a volume that for simplicity is approximated as a spherical shell with a radius of $R_{\mathrm{IR}}=3.54 \times$ $10^{18}\left(L_{\text {disk }} / 10^{45}\right)^{0.5} \mathrm{~cm}$ (Nenkova et al. 2008). The accretion disk luminosity was estimated using the Swift UVOT data points observed during the flaring periods. Reproducing the UVOT fluxes with the Shakura-Sunyaev disk spectrum (Shakura \& Sunyaev 1973) fixing the peak energy at $\sim 10 \mathrm{eV}$, we obtained that the disk luminosity $L_{\text {disk }}=1.2 \times 10^{45} \mathrm{erg} \mathrm{s}^{-1}$ (see Fig. 2 black dashed line), which is close to the value obtained in Janiak et al. (2016).

\subsection{Large-scale jet emission}

We assembled the 3C 120 knot SEDs from the radio to X-ray bands, using the radio to optical data from Harris et al. (2004) (black points in Fig. 3) and the X-ray data obtained in Sect. 3 (red points in Fig. 3). The X-ray flux is well above the extrapolation from the radio to optical spectra and it hardens; this is more evident for the $\mathrm{k} 25$ new where $L_{\mathrm{X}} / L_{\mathrm{rad}} \simeq 250$. This confirms the previous findings that two different components are necessary to explain the radio to optical and X-ray emission from the knots of 3 C 120.

The detected highly polarized emission led to a conclusion that the radio to optical emission from the knots is of a synchrotron origin. The radiative mechanism usually considered to explain the origin of the X-ray emission is either the synchrotron emission from a second, much more energetic population of electrons (e.g., Jester et al. 2006; Harris et al. 2004; Marshall et al. 2002; Röser et al. 2000) or the inverse Compton scattering on various possible sources of soft photons, including the synchrotron photons (SSC; e.g., Hardcastle et al. 2002) and the cosmic microwave background photons (IC/CMB: e.g., Tavecchio et al. 2000). Most naturally, the X-ray emission could originate from IC scattering of synchrotron photons with a density of $U_{\text {syn }}=L_{\text {radio }} / 4 \pi R_{\mathrm{k} 4}^{2} c \approx 3.2 \times 10^{-14} \mathrm{erg} \mathrm{cm}^{-3}$ where $L_{\text {radio }} \simeq 2.0 \times 10^{40} \mathrm{erg} \mathrm{s}^{-1}$ is the radio luminosity of $\mathrm{k} 4$ and $R_{\mathrm{k} 4} \approx 0.42 \mathrm{kpc}$ is the knot size. When comparing the radio and X-ray data it becomes clear that $L_{\mathrm{SSC}} \geq L_{\mathrm{syn}}\left(U_{\mathrm{B}} \leq U_{\text {syn }}\right)$ is satisfied only if $B \leq 0.8 \mu \mathrm{G}$, which is in contradiction with the averaged value of $\geq 10 \mu \mathrm{G}$ usually estimated for the knots. In such a weak magnetic field, the observed radio luminosity can be accounted for only if the particle energy $\left(U_{\mathrm{e}}\right)$ strongly dominates over the magnetic field, thus contravening the equipartition condition, which means that we only consider the inverse Compton scattering of $\mathrm{CMB}$ photons and synchrotron emission from a second, much more energetic population of electrons. In the IC/CMB scenario, it is assumed that the emitting region moves with a relativistic bulk Lorentz factor of $\Gamma_{\text {bulk }}$ in order to predict a larger X-ray luminosity since in the jet frame the energy density of CMB photons will be enhanced by a factor of $\Gamma_{\text {bulk }}^{2}$. The condition of $L_{\mathrm{X} \text {-ray }}>L_{\text {radio }}$ is satisfied only if $\delta$ is at least 10 . In contrast, if the $\mathrm{X}$-ray emission is produced by synchrotron radiation from a second population of relativistic electrons with very high maximum energy, a highly relativistic jet is not required and we assume $\delta=1$. Since the electrons with high maximum energy would quickly cool down, most likely they were produced in a separate episode of acceleration that occurred more recently. Moreover, the second population of electrons can be produced in different parts of the knot (e.g., Ostrowski 2000; Stawarz \& Ostrowski 2002). We do not go into much detail, but we assume that there are two different electron populations responsible for the emission. 
Table 2. Parameters obtained from the fit of the emission from the inner jet of $3 \mathrm{C} 120$ during quiescent and flaring states.

\begin{tabular}{lccccc}
\hline \hline & Parameter & SSC (blue-dashed) & SSC (red) & SSC & SSC+EIC \\
\hline Doppler factor & $\delta$ & 4 & 4 & 6 & $4(6)$ \\
Normalization of electron distribution & $N_{0}^{\prime} \times 10^{50} \mathrm{eV}^{-1}$ & $15292.18_{-10357.06}^{+34383.07}$ & $16.77_{-14.02}^{+176.69}$ & $1.96_{-1.13}^{+2.75}$ & $1713.92_{-18.69}^{+19.88}\left(817.55_{-117.96}^{+118.64}\right)$ \\
Electron spectral index & $\alpha$ & $3.12_{-0.16}^{+0.15}$ & $1.85_{-0.22}^{+0.31}$ & $2.79_{-0.13}^{+0.16}$ & $3.12 \pm 0.22\left(3.24_{-0.80}^{+0.89}\right)$ \\
Minimum electron energy & $E_{\min }^{\prime}(\mathrm{MeV})$ & $354.51_{-24.55}^{+27.20}$ & $228.18_{-120.10}^{+92.19}$ & $67.57_{-20.65}^{+18.03}$ & $117.23_{-12.64}^{+11.80}\left(514.51_{-352.58}^{+569.19}\right)$ \\
Cutoff electron energy & $E_{\mathrm{cut}}^{\prime}(\mathrm{GeV})$ & $3.21_{-0.46}^{+0.60}$ & $4.61_{-0.77}^{+1.63}$ & $6.32_{-1.48}^{+2.93}$ & $1.68_{-0.41}^{+0.42}\left(4.05_{-2.10}^{+5.37}\right)$ \\
Maximum electron energy & $E_{\max }^{\prime}(\mathrm{TeV})$ & $2.30_{-1.54}^{+3.84}$ & $1.90_{-1.26}^{+3.02}$ & $1.83_{-1.27}^{+2.19}$ & $10.71_{-6.56}^{+4.64}\left(54.56_{-40.27}^{+75.36}\right)$ \\
Magnetic field & $\mathrm{B}[\mathrm{G}]$ & $0.16_{-0.007}^{+0.006}$ & $0.0023_{-0.0000}^{+0.0002}$ & $0.86_{-0.09}^{+0.11}$ & $0.63 \pm 0.12\left(0.11_{-0.08}^{+0.11}\right)$ \\
Electron energy density & $U_{\mathrm{e}}\left(\mathrm{erg} \mathrm{cm}^{-3}\right)$ & $1.02 \times 10^{-3}$ & $1.99 \times 10^{-2}$ & 0.14 & $4.39 \times 10^{-4}(0.25)$ \\
Jet power in magnetic field & $L_{\mathrm{B}} \times 10^{44} \mathrm{erg} \mathrm{s}^{-1}$ & 2.58 & $4.98 \times 10^{-4}$ & 0.22 & $38.34(0.0034)$ \\
Jet power in electrons & $L_{\mathrm{e}} \times 10^{44} \mathrm{erg} \mathrm{s}^{-1}$ & 2.46 & 48.00 & 1.09 & $1.06(2.02)$ \\
\hline
\end{tabular}

In the IC/CMB scenario, the underlying electron distribution is given by Eq. (1) with the cutoff energy allowed to vary only for $\mathrm{k} 4$, where the optical flux at $\sim 10^{14} \mathrm{~Hz}$ allows the $\mathrm{HE}$ tail of synchrotron emission to be constrained, while for the other knots it is fixed at $E_{\text {cut }}=100 \mathrm{GeV}$ due to the lack of data. In addition, a power-law distribution in the form of $N_{\mathrm{PL}} \sim E_{\mathrm{e}}^{-\alpha_{\mathrm{PL}}}$ is added to model the X-ray spectrum in the two-component synchrotron scenario. Since there are not enough data to constrain $E_{\max , \mathrm{PL}}$, an artificial $\mathrm{HE}$ limit of $E_{\mathrm{PL} \text {, max }}=1 \mathrm{PeV}$ has been introduced, whereas $E_{\mathrm{PL}, \min }$ is left as a free parameter with the restriction that the flux from the second component should not exceed the first. In our calculations we used the shapes and sizes of the knots as provided in Harris et al. (2004). To make the deviation from the equipartition condition as small as possible, we define $\eta=$ $U_{\mathrm{e}} / U_{\mathrm{B}}$, which is used as a free parameter with $U_{\mathrm{e}}$ during the fit. This will allow us to find the optimum value for $\eta$ when $\eta=1$ does not give satisfactory representation of the data. In the twocomponent synchrotron model, we fix $\eta=1$ and perform fitting of the radio to optical data. Then fixing this magnetic field, the $\mathrm{X}$-ray data are fitted with the second component.

\subsection{Fitting technique}

In order to constrain the model's free parameters we have modified the naima package (Zabalza 2015) which derives the bestfit and uncertainty distributions of spectral model parameters through Markov chain Monte Carlo (MCMC) sampling of their likelihood distributions. The prior likelihood, our prior knowledge of the probability distribution of a given model parameter, and the data likelihood functions are passed onto the emcee sampler function for an affine-invariant MCMC run. In the parameter sampling, the expected ranges considered are $1.5 \leq$ $\left(\alpha, \alpha_{\mathrm{PL}}\right) \leq 10$ and $0.511 \mathrm{MeV} \leq E_{\text {cut }}^{\prime} \leq 1 \mathrm{TeV}$, and $N_{0}$ and $B$ are defined as positive parameters. The synchrotron emission is calculated using the parameterization of the emissivity function of synchrotron radiation in random magnetic fields presented in Aharonian et al. (2010), while the IC emission is computed based on the monochromatic differential cross section of Aharonian \& Atoyan (1981).

\section{Results and discussion}

In this paper, the multiwavelength emission from the 3C 120 core is investigated using Swift XRT/UVOT and Fermi LAT data. Quiescent and flaring states are identified and their modeling allowed us to investigate the jet properties and physical processes that take place in the core where, most likely, the jet is formed.
On the other hand, the jet properties are also estimated at large distances from the core using the Chandra X-ray data.

The adaptively binned $\gamma$-ray light curve showed that before MJD 56900 and after MJD 57300, the source was in a quiescent state characterized by a relatively faint $\gamma$-ray emission with the flux and the photon index consistent with the previously reported values. Then, from MJD 56900 to MJD 57 300, most of the time, the source was in an effective $\gamma$-ray emitting state with rapid $\gamma$-ray flares. During the bright periods, the $\gamma$-ray photon index hardened and corresponded to $\approx 2.3$ and $\approx 2.0$. Two strong events with $(7.46 \pm 1.56) \times 10^{-6}$ photon $\mathrm{cm}^{-2} \mathrm{~s}^{-1}$ and $(4.71 \pm 0.92) \times 10^{-6}$ photon $\mathrm{cm}^{-2} \mathrm{~s}^{-1}$ within accordingly $19.0 \mathrm{~min}$ and $3.15 \mathrm{~h}$ were detected on April 24, 2015, which are the highest fluxes detected so far from 3C 120. At a distance of $144.9 \mathrm{Mpc}$, these correspond to an isotropic $\gamma$-ray luminosity of $(1.20-1.66) \times 10^{46} \mathrm{erg} \mathrm{s}^{-1}$. The same value estimated for the quiescent state (the first bin in Fig. 1 red data) is $2.85 \times 10^{43} \mathrm{erg} \mathrm{s}^{-1}$. Yet, assuming $\delta=6$, the total power emitted in the $\gamma$-ray band in the proper frame of the jet would be $L_{\mathrm{em}, \gamma}=L_{\gamma} / 2 \delta^{2}=(1.67-2.31) \times 10^{44} \mathrm{erg} \mathrm{s}^{-1}$ during the peak flux and $L_{\mathrm{em}, \gamma}=8.9 \times 10^{41} \mathrm{erg} \mathrm{s}^{-1}$ in a quiescent state (assuming $\delta=4$ ). Thus, during the peak emission, the energy released in the $\gamma$-ray band corresponds to large fraction of Eddington luminosity $\left(L_{\mathrm{Edd}}=6.5 \times 10^{45} \mathrm{erg} \mathrm{s}^{-1}\right.$ for the black hole mass of $5.5 \times 10^{7} M_{\odot}$, Peterson et al. 2004), while it is a small fraction $\left(\sim 1.4 \times 10^{-4}\right)$ in the quiescent state.

Usually the radio galaxies have a luminosity of $\leq 10^{44} \mathrm{erg} \mathrm{s}^{-1}$ (Abdo et al. 2010a; Ackermann et al. 2015), and the peak $\gamma$-ray apparent luminosity of $(1.20-1.66) \times 10^{46} \mathrm{erg} \mathrm{s}^{-1}$ is unusual, more characteristic for BL Lac objects. Such a strong $\gamma$-ray output observed from 3C 120 is not surprising as the jet inclination angle is relatively small compared with other radio galaxies.

In the X-ray band, the average flux in the $0.5-10.0 \mathrm{keV}$ range is around $(2.4-4.3) \times 10^{-11} \mathrm{erg} \mathrm{cm}^{-2} \mathrm{~s}^{-1}$ in the quiet state and $(5.4-6.7) \times 10^{-11} \mathrm{erg} \mathrm{cm}^{-2} \mathrm{~s}^{-1}$ on October 4-7, 2014 (active state). When the lowest and highest fluxes from Table 1 are compared, a nearly 2.8 times increase of the X-ray flux is found, but its amplitude is lower than the substantial increase in the $\gamma$-ray band. During the considered periods, the source spectra were always hard, $\Gamma_{\mathrm{X}}<2.0$. At bright $\gamma$-ray flares, the $\mathrm{X}$-ray photon index softened (1.72-1.80) as compared with the hard photon index of $1.42-1.53$ in a quiescent state. The small change in the $\mathrm{X}$-ray flux level and the photon index softening might indicate that different mechanisms are contributing to the acceleration and/or cooling of electrons, which modifies the power-law index and the minimum energy of underlying electrons. 
The data accumulated during several observations of 3C 120 allowed us to resolve and study the X-ray emission from the large-scale jet of 3C 120. In particular, the counts from $\mathrm{k} 4$ and k25 are high enough for detailed spectral analysis, resulting in a flux of $\leq 1.60 \times 10^{-13} \mathrm{erg} \mathrm{cm}^{-2} \mathrm{~s}^{-1}$ with hard X-ray photon indices $\leq 1.8$, which implies that most of the energy is released above $10 \mathrm{keV}$. It is interesting to note that the X-ray flux of $(1.22 \pm 0.19) \times 10^{-13} \mathrm{erg} \mathrm{cm}^{-2} \mathrm{~s}^{-1}$ from $\mathrm{k} 25$ outer located at $\sim 16 \mathrm{kpc}$ from the core is of the order of the flux from the nearby $\mathrm{k} 4$ at $\sim 2.5 \mathrm{kpc}$, however with a much harder X-ray photon index: $\Gamma_{\mathrm{X}}=1.62 \pm 0.11$.

\subsection{Origin of emission from the inner jet}

The broadband emission modeling results obtained in the quiescent and flaring states are shown in Fig. 2 with the corresponding parameters in Table 2 . In the quiescent state, the X-ray spectra have different photon indices, $\Gamma_{X}=1.42 \pm 0.07$ and $1.53 \pm 0.08$, and is the reason why both spectra have been considered during the fit. In the fit we also included the archival radio data from the observations in the period when the source was in the quiescent state. The radio emission can be produced from the low energy electrons which are accumulated for longer periods, so the radio flux should not exceed the presented limit. When both radio and optical/UV data are considered, a larger value of $E_{\min }^{\prime}$ is required in order to have model which predicts emission below the radio flux. However, the increase of $E_{\min }^{\prime}$ would also affect the flux predicted by SSC in the X-ray band; for example, the blue dashed line in Fig. 2 illustrates the model for $E_{\text {min }}^{\prime} \simeq 354.51 \pm 25.91 \mathrm{MeV}$ (summing the errors in quadrature) beyond which the model predicts either a high radio flux or a low X-ray flux. The magnetic field is $B=0.16 \mathrm{G}$ with an energy density of $U_{\mathrm{B}}=1.07 \times 10^{-3} \mathrm{erg} \mathrm{cm}^{-3}$, slightly higher than that of the electrons, $U_{\mathrm{e}}=1.02 \times 10^{-3} \mathrm{erg} \mathrm{cm}^{-3}$. This magnetic field energy density should be considered as an upper limit since the Swift UVOT data may represent the direct thermal disk emission, which means, in principle, that the synchrotron component can be much lower. Thus, in the second modeling, we assume that the low energy component is only defined by the radio data (blue and red solid lines in Fig. 2). In this case the underlying electron distribution is characterized by a harder power-law in$\operatorname{dex}(\alpha=2.22 \pm 0.19$ and $\alpha=1.85 \pm 0.27$ for blue and red solid lines, respectively) and higher cutoff energy $E_{\text {cut }}^{\prime}=4.61 \pm 1.27$. The magnetic field is significantly lower, $B=2.3 \times 10^{-3} \mathrm{G}$, and the jet should be strongly particle-dominated to have the peak flux of the HE component exceeding that of the low energy one. This particle dominance can be minimized assuming that the X-ray emission is of a different origin (e.g., from another blob or from thermal Comptonization near the disk). When the optical/UV and X-ray data are assumed as upper limits in the fit, a lower flux from SSC emission is expected (gray line in Fig. 2, left panel) and now $U_{\mathrm{e}} / U_{\mathrm{B}} \approx 43$.

When the SED in the flaring period is modeled considering SSC emission (red solid line in Fig. 2, right panel) the electron distribution and the magnetic field should vary. As the X-ray spectrum is soft $\left(\Gamma_{\mathrm{X}}=1.8\right)$, the modeling yielded a lower $E_{\text {min }}^{\prime}=(67.57 \pm 19.38) \mathrm{MeV}$ and $\alpha \simeq 2.79 \pm 0.15$. As the $\gamma$-ray spectrum is shifted to higher energies, then a cutoff of $E_{\text {cut }}^{\prime}=(6.32 \pm 2.32) \mathrm{GeV}$ is required. Since the emitting region radius decreases by $\sim 25.6$ times, the magnetic field should increase $(0.86 \pm 0.1 \mathrm{G})$ in order to produce a synchrotron flux of the same order (or higher) because the synchrotron emission depends on the total number of the emitting electrons $\left(N_{\mathrm{e}}, \delta\right.$, and magnetic field $B$ ). In this case, the required electron energy density exceeds that of the magnetic field only 2.6 times, meaning there is no significant deviation from equipartition. The radio data are also plotted as reference values, but we note that in the flaring state the radio flux can also increase. However, the model does not predict a flux that significantly exceeds the observed radio data.

In the right panel of Fig. 2, SSC (blue dashed line) and EIC (blue dot dashed line) modeling of the SED is shown (SSC+EIC, blue solid line). The X-ray emission can be explained by the SSC emission produced in a blob of a size similar to that emitting in a quiescent state, but additional changes in $\alpha, E_{\text {cut }}^{\prime}$, and $B$ are necessary to account for the new X-ray spectrum. Instead, the $\gamma$-ray emission is entirely due to the IC scattering of external photons in the fast and compact blob, which is strongly particle-dominated with $U_{\mathrm{e}} / U_{\mathrm{B}} \approx 519$, and the magnetic field $B=0.11 \pm 0.1 \mathrm{G}$, which does not differ much from the values obtained in the one-zone models. In the radio band, the modeling predicts a higher flux than the presented radio data. As the radio data are not from the source active periods, this is not a strong argument to disfavor such modeling. When a larger value for $E_{\min }^{\prime}$ is used in the modeling, it does not introduce significant changes in the model parameters (especially in the energetics of the jet). Even if there are not enough data to estimate the parameters with a high significance, which means that the conclusions are not definite, this is an interesting modeling as it could possibility explain the rapid $\gamma$-ray activities. Such a blob can be naturally formed in the reconnection events that could produce compact regions of rapidly moving plasma inside the jet ("jetin-jet scenario", Giannios et al. 2009, 2010).

\subsection{Origin of emission from knots}

The black lines in Fig. 3 represents the IC/CMB radiation model calculated for $\delta=10$. The obtained parameters are presented in Table 3. Similar photon indices observed in the radio and $\mathrm{X}$-ray bands allowed to define the power-law index of electrons which varies from 2.4 to 2.6. The cutoff energy estimated for $\mathrm{k} 4$ is $E_{\mathrm{c}}^{\prime} \approx(916.3 \pm 251.4) \mathrm{GeV}$ implies there is an effective particle acceleration above the $\mathrm{TeV}$ energies. The value of $E_{\min }^{\prime} \simeq(18.20-24.01) \mathrm{MeV}$ is estimated, which we obtain by requiring a turnover below the X-ray data in order not to overproduce the radio/optical flux, but in principle lower values cannot be excluded. The IC scattering of CMB photons with $u_{\mathrm{CMB}} \simeq 4.0 \times 10^{-11} \mathrm{erg} \mathrm{cm}^{-3}$ density (when $\delta=10$ ) still predicts a flux lower than the observed value, so we were forced to adopt larger values of $\eta$. We found that when $B=(0.93-5.6) \mu \mathrm{G}$ and $\eta=(83.4-410.8)$, the IC/CMB model can reproduce the observed spectra. The maximum electron energy density is estimated to be $1.12 \times 10^{-10} \mathrm{erg} \mathrm{cm}^{-3}$ for the $\mathrm{k} 4$, while for the other knots it is $>4$ times lower. This is natural, since even if the total number of particles is conserved, the low energy cutoff moves to lower energies (because of adiabatic losses), the normalization decreases and $U_{\mathrm{e}}$ does so as well. The IC/CMB component predicts emission up to $\sim \epsilon_{\mathrm{CMB}}\left(E_{\mathrm{e}} / m_{\mathrm{e}} c^{2}\right)^{2} \approx 27.4 \mathrm{GeV}$ so that $\gamma$-ray emission is also expected. However, even if the predicted flux is above the Fermi LAT sensitivity $\left(\sim 10^{-13} \mathrm{erg} \mathrm{cm}^{-2} \mathrm{~s}^{-1}\right)$, its level (a few times $10^{-13} \mathrm{erg} \mathrm{cm}^{-2} \mathrm{~s}^{-1}$ ) would be still below the core emission in the quiet state (Fig. 2). Moreover, the Doppler boosting of $\delta \geq 10$ requires the jet to be highly relativistic or viewed at small angles at kpc distances from the core, which seems unrealistic for 3C 120, so even lower flux levels are expected.

The blue lines in Fig. 3 show the two-component synchrotron model fitting of the knot SEDs. The radio to optical data of the 
Table 3. Parameters derived from modeling the knot SEDs.

\begin{tabular}{lcccc}
\hline \hline Parameter & $\mathrm{k} 4$ & $\mathrm{k} 25$ inner & $\mathrm{k} 25$ outer & $\mathrm{k} 25$ new \\
\hline$U_{\mathrm{e}}^{\prime}\left(U_{\mathrm{e}}\right)\left[\mathrm{erg} \mathrm{cm}^{-3}\right] \times 10^{-12}$ & $111.8_{-9.91}^{+16.49}\left(531.52_{-84.76}^{+110.7}\right)$ & $20.65_{-3.47}^{+9.95}\left(102.91_{-4.22}^{+82.45}\right)$ & $30.25_{-2.73}^{+6.21}\left(48.56_{-13.47}^{+26.11}\right)$ & $7.43_{-1.43}^{+2.89}\left(4.09_{-0.63}^{+1.27}\right)$ \\
$\alpha$ & $2.59 \pm 0.03(2.48 \pm 0.06)$ & $2.44 \pm 0.1\left(2.41_{-0.26}^{+0.22}\right)$ & $2.42 \pm 0.04\left(2.20_{-0.15}^{+0.17}\right)$ & $2.62_{-0.10}^{+0.14}(2)$ \\
$E_{\min }^{\prime}\left(E_{\min }\right)[\mathrm{MeV}]$ & $24.01_{-5.44}^{+3.62}\left(4.40_{-2.31}^{+2.92}\right)$ & $18.2 \pm 10.6\left(3.39_{-1.90}^{+3.57}\right)$ & $22.23_{-8.43}^{+5.53}\left(4.04_{-2.92}^{+8.48}\right)$ & $19.1_{-8.57}^{+7.11}\left(1445.67_{-1198.91}^{+3852.81}\right)$ \\
$B^{\prime}(B)[\mu \mathrm{G}]$ & $5.61(115.6)$ & $2.35(50.85)$ & $1.36(34.94)$ & $0.93(10.14)$ \\
$\eta$ & $89.39_{-12.75}^{+7.48}$ & $94.04_{-43.7}^{+49.8}$ & $410.86_{-104.94}^{+64.28}$ & $214.3_{-124.63}^{+162.67}$ \\
$U_{\mathrm{e}, \mathrm{p}}\left(\mathrm{erg} \mathrm{cm}^{-3}\right) \times 10^{-14}$ & $6.01_{-2.38}^{+5.85}$ & $2.36_{-1.19}^{+2.59}$ & $7.22_{-1.93}^{+3.01}$ & $13.22_{-5.62}^{+15.7}$ \\
$\alpha_{\mathrm{p}}$ & $2.69_{-0.18}^{+0.22}$ & $2.66_{-0.54}^{+1.15}$ & $2.32_{-0.18}^{+0.17}$ & $2.73_{-0.24}^{+0.29}$ \\
$E_{\min , \mathrm{p}}[\mathrm{TeV}]$ & $2.83_{-1.73}^{+3.22}$ & $6.89_{-5.17}^{+9.83}$ & $5.31_{-3.16}^{+5.65}$ & $8.88_{-6.78}^{+10.24}$ \\
$L_{\mathrm{B}} \times 10^{42}$ & $6.48(128)$ & $71.26(333.7)$ & $31.98(211)$ & $0.67(3.69)$ \\
$L_{\mathrm{e}} \times 10^{44}$ & $5.81(1.28)$ & $66.98(3.34)$ & $131.45(2.11)$ & $1.44(0.04)$ \\
\hline
\end{tabular}

four knots are modeled with synchrotron emission with the following plausible parameters: $B$ between 10.1 and $115.6 \mu \mathrm{G}$, and an electron power-law index of $\alpha=2.20-2.48$. The plasma in the knots is in equipartition, $U_{\mathrm{e}}=U_{\mathrm{B}}=(4.1-531.5) \times$ $10^{-12} \mathrm{erg} \mathrm{cm}^{-3}$, which requires a magnetic field that is more than 10 times stronger for all the knots with respect to the previous modeling. The synchrotron emission of the second population of electrons for the same magnetic field can explain the X-ray flux when $E_{\min } \simeq(2.83-8.88) \mathrm{TeV}$ and $\alpha=2.32-2.69$. The particle energy density of this component is negligible compared with the other one. A significant contribution from the electrons with $E_{\mathrm{e}} \simeq 10 \mathrm{TeV}$ is expected, the cooling time of which $t_{\text {cool }}=6 \pi m_{\mathrm{e}}^{2} c^{3} / \sigma_{\mathrm{T}} B^{2} E_{\mathrm{e}} \simeq 255.75 \mathrm{yr}$. This corresponds to a travel distance of $c \times t_{\text {cool }} \simeq 78.4 \mathrm{pc}$, which is much smaller than the size of the knots. Thus, it is required that the particle acceleration in situ over the entire volume of the knots should be extremely efficient.

The above obtained parameters were estimated taking into account the equipartition condition when the system is close to internal pressure or energy density condition, but for jet dynamics and propagation the jet pressure balance with the ambient medium is more important. The results presented here and previous observations of the knots allow us to put important constraints on some of the physical parameters of the jet. The jet half opening angle $\left(\theta_{\mathrm{j}}\right)$ at kpc scale can be estimated using the first resolved jet knot (k4); at a distance of 4 arcseconds from the core its radius is 0.738 arcsecond, implying $\theta_{\mathrm{j}} \simeq 10.45^{\circ}$. Having the independent information on the jet Doppler factor, the upper bound on the magnetosonic (Mach) number is $M_{\mathrm{j}} \sim 1 / \tan \left(\theta_{\mathrm{j}}\right) \Gamma_{\text {bulk }} \simeq 5.42 / \Gamma_{\text {bulk }}$. If the jet remains relativistic up to kpc scale with $\Gamma_{\text {bulk }}=5.3 \pm 1.2$, then $M_{\mathrm{j}} \simeq 1.02$. For the pc jet of $3 \mathrm{C} 120$, assuming an $R=1.56 \times 10^{16} \mathrm{~cm}$ emitting region at parsec distance, $M_{\mathrm{j}}$ corresponds to 37.32 . Thus, the relativistic jet with an initial high Mach number comes into static pressure equilibrium with the interstellar medium of the parent galaxy, starting to interact with it, causing the Mach number to decrease. This is qualitatively supported by the radio/X-ray observations, which reveal that at a distance of $\mathrm{k} 4 / \mathrm{k} 7$ knots the jet starts to expand (e.g., Fig. 3 of Harris et al. 2004).

\subsection{Jet energetics}

The fundamental quantity is the total power (particles + magnetic field) transported by the jet flow. The total jet power can be estimated using the parameters derived from the SED modeling using $L_{\mathrm{e}}=\pi c R_{\mathrm{b}}^{2} \Gamma^{2} U_{\mathrm{e}}$ and $L_{\mathrm{B}}=\pi c R_{\mathrm{b}}^{2} \Gamma^{2} U_{\mathrm{B}}$ (Celotti \& Ghisellini 2008) for electrons and magnetic field, respectively ( $\Gamma=1$ is assumed in the two-component synchrotron model). The protons with unknown contribution to the jet have not been considered in the calculations since a number of assumptions need to be made.

In the quiescent state, the total power at the jet core for all models presented in Fig. 2 is $L_{\text {jet }}=L_{\mathrm{e}}+L_{\mathrm{B}} \simeq$ (2.35-48.0) $\times 10^{44} \mathrm{erg} \mathrm{s}^{-1}$ (as in Tanaka et al. 2015; Janiak et al. 2016; Sahakyan et al. 2015). Thus, the isotropic $\gamma$-ray luminosity, $L_{\mathrm{em}, \gamma} \simeq 8.2 \times 10^{41} \mathrm{erg} \mathrm{s}^{-1}$, is only a small fraction of the total jet power. The jet's total power, $L_{\text {jet }} \simeq 1.31 \times 10^{44} \mathrm{erg} \mathrm{s}^{-1}$, decreases in the active state, since it scales with the emitting region size $\left(L_{\text {jet }} \sim R^{2} U\right)$ and a smaller region is considered. However, this region is more energetic; the particle energy density is $\sim 146.9$ times higher than that in the quiet state. The SSC+EIC scenario requires a total jet luminosity of $L_{\text {jet }} \simeq$ $4.14 \times 10^{45} \mathrm{erg} \mathrm{s}^{-1}$, which is higher than the previous values, but is easily achievable for the black hole mass in 3C 120 .

When the jet power is estimated for the knots, their largest reasonable volumes are used, so that the obtained values are the upper limits. In case of the beamed IC/CMB scenario the total jet power should be $L_{\text {jet }} \simeq(1.4-131.4) \times 10^{44} \mathrm{erg} \mathrm{s}^{-1}$ in order to explain the X-ray luminosity of $L_{\mathrm{X}} \simeq(1.0-4.0) \times 10^{41} \mathrm{erg} \mathrm{s}^{-1}$. This jet luminosity is mostly defined by the kinetic energy of particles since the modeling reveals a moderate domination of particles over the magnetic field $(\eta \gg 1)$. In the two-component synchrotron model, the total jet luminosity is lower, $L_{\text {jet }} \leq$ $6.7 \times 10^{44} \mathrm{erg} \mathrm{s}^{-1}$, where the contribution of the X-ray emitting component is negligible. The powers independently derived for the inner and outer regions of the jet are of the same order, suggesting that the jet does not substantially dissipate its power until its end, but becomes radiatively inefficient farther from the formation point.

\section{Summary}

The main properties of the powerful jet of 3C 120 are investigated by comparing the physical state of the plasma on sub-pc and $\mathrm{kpc}$ scales. The main processes responsible for the broadband emission in the innermost $(\leq \mathrm{pc} ;$ Swift XRT/UVOT and Fermi LAT data) and outer ( $\geq 1 \mathrm{kpc}$; Chandra data) regions are also studied.

On April 24, 2015, a rapid and dramatic increase in the $\gamma$-ray flux was observed from the inner jet of 3C 120. Within 
$19.0 \mathrm{~min}$ and $3.15 \mathrm{~h}$ the flux was as high as $(7.46 \pm 1.56) \times$ $10^{-6}$ photon $\mathrm{cm}^{-2} \mathrm{~s}^{-1}$ and $(4.71 \pm 0.92) \times 10^{-6}$ photon $\mathrm{cm}^{-2} \mathrm{~s}^{-1}$ above $100 \mathrm{MeV}$ which corresponds to an isotropic $\gamma$-ray luminosity of $(1.2-1.6) \times 10^{46} \mathrm{erg} \mathrm{s}^{-1}$. A luminosity of this level is unusual for radio galaxies and more typical for BL Lacs. The synchrotron/SSC mechanism gives a reasonable explanation of the multiwavelength SED in the quiescent and flaring states. The increase and rapid changes in the flaring state can be also explained assuming an additional contribution from the blob where the dominant photon fields are of external origin. The necessary jet kinetic power is $L_{\mathrm{jet}} \simeq(1.31-48.0) \times 10^{44} \mathrm{erg} \mathrm{s}^{-1}$.

The X-ray emission from the knots has a hard photon index of $\simeq(1.6-1.8)$ with a luminosity of $L_{X} \simeq(1.0-4.01) \times$ $10^{41} \mathrm{erg} \mathrm{s}^{-1}$. This X-ray emission can be explained by IC/CMB models only if $\delta>10$, otherwise the particle energy density will strongly dominate over that of the magnetic field. If the X-rays were produced from the direct synchrotron radiation of the second population of electrons, which were produced more recently than the cooler population responsible for the radio to optical spectrum, then a lower jet luminosity and no bulk relativistic motion on $\mathrm{kpc}$ scales is required.

The jet luminosities of the innermost and outer regions are comparable, suggesting that the jet does not suffer important energy losses when propagates from the regions close to the black hole to those at hundreds of kiloparsecs from it. However, at larger distances the magnetic field and the particle energy density decrease and the jet becomes radiatively inefficient.

Acknowledgements. This work was supported by the RAMES State Committee of Science, within the frame of the research project No. 15T-1C375. Also, this work was made in part by a research grant from the Armenian National Science and Education Fund (ANSEF) based in New York, USA.

\section{References}

Abdo, A. A., Ackermann, M., Ajello, M., et al. 2009, ApJ, 707, 55 Abdo, A. A., Ackermann, M., Ajello, M., et al. 2010a, ApJ, 720, 912 Abdo, A. A., Ackermann, M., Ajello, M., et al. 2010b, ApJ, 719, 1433 Acero, F., Ackermann, M., Ajello, M., et al. 2015, ApJS, 218, 23 Ackermann, M., Ajello, M., Atwood, W. B., et al. 2015, ApJ, 810, 14 Aharonian, F. A. 2002, MNRAS, 332, 215

Aharonian, F. A., \& Atoyan, A. M. 1981, Ap\&SS, 79, 321

Aharonian, F. A., Kelner, S. R., \& Prosekin, A. Y. 2010, Phys. Rev. D, 82, 043002 Atwood, W. B., Abdo, A. A., Ackermann, M., et al. 2009, ApJ, 697, 1071

Błażejowski, M., Sikora, M., Moderski, R., \& Madejski, G. M. 2000, ApJ, 545, 107

Bloom, S. D., \& Marscher, A. P. 1996, ApJ, 461, 657

Breeveld, A. A., Landsman, W., Holland, S. T., et al. 2011, in AIP Conf. Ser. 1358, eds. J. E. McEnery, J. L. Racusin, \& N. Gehrels, 373

Casadio, C., Gómez, J. L., Grandi, P., et al. 2015, ApJ, 808, 162
Celotti, A., \& Ghisellini, G. 2008, MNRAS, 385, 283 Fanaroff, B. L., \& Riley, J. M. 1974, MNRAS, 167, 31P Gehrels, N., Chincarini, G., Giommi, P., et al. 2004, ApJ, 611, 1005 Ghisellini, G., \& Tavecchio, F. 2009, MNRAS, 397, 985

Ghisellini, G., Maraschi, L., \& Treves, A. 1985, A\&A, 146, 204

Ghisellini, G., Foschini, L., Volonteri, M., et al. 2009a, MNRAS, 399, L24

Ghisellini, G., Maraschi, L., \& Tavecchio, F. 2009b, MNRAS, 396, L105

Ghisellini, G., Tagliaferri, G., Foschini, L., et al. 2011, MNRAS, 411, 901

Giannios, D., Uzdensky, D. A., \& Begelman, M. C. 2009, MNRAS, 395, L29

Giannios, D., Uzdensky, D. A., \& Begelman, M. C. 2010, MNRAS, 402, 1649

Giommi, P., Polenta, G., Lähteenmäki, A., et al. 2012, A\&A, 541, A160

Gómez, J.-L., Marscher, A. P., Alberdi, A., Martí, J. M., \& Ibáñez, J. M. 1998, ApJ, 499, 221

Gómez, J.-L., Marscher, A. P., \& Alberdi, A. 1999, ApJ, 521, L29

Halpern, J. P. 1985, ApJ, 290, 130

Hardcastle, M. J., Birkinshaw, M., Cameron, R. A., et al. 2002, ApJ, 581, 948

Harris, D. E., Hjorth, J., Sadun, A. C., Silverman, J. D., \& Vestergaard, M. 1999, ApJ, 518, 213

Harris, D. E., Mossman, A. E., \& Walker, R. C. 2004, ApJ, 615, 161

Hjorth, J., Vestergaard, M., Sorensen, A. N., \& Grundahl, F. 1995, ApJ, 452, L17

Homan, D. C., Ojha, R., Wardle, J. F. C., et al. 2001, ApJ, 549, 840

Hovatta, T., Valtaoja, E., Tornikoski, M., \& Lähteenmäki, A. 2009, A\&A, 494, 527

Inoue, S., \& Takahara, F. 1996, ApJ, 463, 555

Janiak, M., Sikora, M., \& Moderski, R. 2016, MNRAS, 458, 2360

Jester, S., Harris, D. E., Marshall, H. L., \& Meisenheimer, K. 2006, ApJ, 648, 900

Jorstad, S. G., Marscher, A. P., Lister, M. L., et al. 2005, AJ, 130, 1418

Kataoka, J., Stawarz, Ł., Takahashi, Y., et al. 2011, ApJ, 740, 29

Lott, B., Escande, L., Larsson, S., \& Ballet, J. 2012, A\&A, 544, A6

Maraschi, L., Ghisellini, G., \& Celotti, A. 1992, ApJ, 397, L5

Marscher, A. P., Jorstad, S. G., Gómez, J.-L., et al. 2002, Nature, 417, 625

Marshall, H. L., Miller, B. P., Davis, D. S., et al. 2002, ApJ, 564, 683

Nenkova, M., Sirocky, M. M., Nikutta, R., Ivezić, Ž., \& Elitzur, M. 2008, ApJ, 685,160

Ostrowski, M. 2000, MNRAS, 312, 579

Paggi, A., Cavaliere, A., Vittorini, V., D’Ammando, F., \& Tavani, M. 2011, ApJ, 736, 128

Peterson, B. M., Ferrarese, L., Gilbert, K. M., et al. 2004, ApJ, 613, 682

Poole, T. S., Breeveld, A. A., Page, M. J., et al. 2008, MNRAS, 383, 627

Pozo Nuñez, F., Haas, M., Ramolla, M., et al. 2014, A\&A, 568, A36

Roming, P. W. A., Koch, T. S., Oates, S. R., et al. 2009, ApJ, 690, 163

Röser, H.-J., Meisenheimer, K., Neumann, M., Conway, R. G., \& Perley, R. A. 2000, A\&A, 360, 99

Sahakyan, N., Zargaryan, D., \& Baghmanyan, V. 2015, A\&A, 574, A88

Schlafly, E. F., \& Finkbeiner, D. P. 2011, ApJ, 737, 103

Shakura, N. I., \& Sunyaev, R. A. 1973, A\&A, 24, 337

Sikora, M., Begelman, M. C., \& Rees, M. J. 1994, ApJ, 421, 153

Stawarz, Ł., \& Ostrowski, M. 2002, ApJ, 578, 763

Tanaka, Y. T., Doi, A., Inoue, Y., et al. 2015, ApJ, 799, L18

Tavecchio, F., Maraschi, L., Sambruna, R. M., \& Urry, C. M. 2000, ApJ, 544, L23

Tavecchio, F., Becerra-Gonzalez, J., Ghisellini, G., et al. 2011, A\&A, 534, A86 Urry, C. M., \& Padovani, P. 1995, PASP, 107, 803

Walker, R. C., Benson, J. M., \& Unwin, S. C. 1987, ApJ, 316, 546

Zabalza, V. 2015, Proc. of the 34th International Cosmic Ray Conference 2015, id. 922

Zhang, J., Bai, J. M., Chen, L., \& Liang, E. 2010, ApJ, 710, 1017 